\title{
Common and distinct regulation of human and mouse brown and beige adipose tissues: a promising therapeutic target for obesity
}

\author{
Xuejiao Liu $^{1}\left(\mathbb{D}\right.$, Christopher Cervantes $^{2}$, Feng Liu ${ }^{1,2 \bowtie}$ (1) \\ ${ }^{1}$ Department of Metabolism and Endocrinology, Metabolic Syndrome Research Center of Central South University, The \\ Second Xiangya Hospital, Central South University, Changsha 410011, China \\ ${ }^{2}$ Department of Pharmacology, University of Texas Health Science Center at San Antonio, San Antonio, TX 78229, USA \\ \ Correspondence: liuf@uthscsa.edu (F. Liu)
}

Received November 10, 2016 Accepted January 26, 2017

\begin{abstract}
Obesity, which underlies various metabolic and cardiovascular diseases, is a growing public health challenge for which established therapies are inadequate. Given the current obesity epidemic, there is a pressing need for more novel therapeutic strategies that will help adult individuals to manage their weight. One promising therapeutic intervention for reducing obesity is to enhance energy expenditure. Investigations into human brown fat and the recently discovered beige/brite fat have galvanized intense research efforts during the past decade because of their pivotal roles in energy dissipation. In this review, we summarize the evolution of human brown adipose tissue (hBAT) research and discuss new in vivo methodologies for evaluating energy expenditure in patients. We highlight the differences between human and mouse BAT by integrating and comparing their cellular morphology, function, and gene expression profiles. Although great advances in hBAT biology have been achieved in the past decade, more cellular models are needed to acquire a better understanding of adipose-specific processes and molecular mechanisms. Thus, this review also describes the development of a human brown fat cell line, which could provide promising mechanistic insights into hBAT function, signal transduction, and development. Finally, we focus on the therapeutic potential and current limitations of hBAT as an anti-glycemic, anti-lipidemic, and weight loss-inducing 'metabolic panacea'.
\end{abstract}

KEYWORDS human brown adipose tissue, energy metabolism, obesity

\section{INTRODUCTION}

Throughout human history, cold weather and famine were threats to survival. To ward off these threats, brown adipose tissue (BAT) and white adipose tissue (WAT) were evolutionarily selected. BAT dissipates energy to keep us warm, while WAT stores excess energy to be spent during times of food deficiency. These two complementary fat depots work to maintain energy balance. However, industrialization and technological advancements in developed societies have broken this balance due to an increase in sedentary occupations, overabundance of food, lack of physical activity, and warm shelter, causing obesity rates to skyrocket as more countries move from being undeveloped to developed. As the World Health Organization (WHO) reported in June 2016, worldwide obesity has more than doubled since 1980 (World Health Organization Fact Sheets: Obesity and Overweight, 2016). In 2014, 39\% of adults aged 18 years and older were overweight, and $13 \%$ of them were obese (World Health Organization Fact Sheets: Obesity and Overweight, 2016). Most of the world's population lives in countries where a high body mass index kills more people than a low one (World Health Organization Fact Sheets: Obesity and Overweight, 2016). In other words, food shortages have not been as deadly as food surpluses. According to the data, obesity, which is associated with type 2 diabetes, polycystic ovarian syndrome, hypertension, cardiovascular disease, etc., is the major public health challenge of the 21 st century (Eckel et al., 2010). Thus, therapeutic approaches that prevent and/or reverse obesity deserve further exploration.

Brown adipocytes, which are rich in mitochondria, are different from white adipocytes because they consume energy more efficiently. Indeed, increased energy expenditure would 
be expected to reverse and/or prevent obesity (Hall et al., 2011). Given that active BAT with energy dissipation function has been confirmed in a number of human adults (Nedergaard et al., 2007; Cypess et al., 2009; Saito et al., 2009; van Marken Lichtenbelt et al., 2009), research interests into human BAT as a means of controlling obesity by diverting excess fat into heat has greatly intensified and represents a novel therapeutic approach for combatting this deadly, metabolic disease.

\section{THE EVOLUTION OF HUMAN BAT RESEARCH}

In 1902, Shinkishi Hatai first reported in a human embryonic development study that BAT is located beneath the sternocleidomastoid and trapezius muscles and runs laterally and parallel to the neck (Hatai, 1902). During human infancy, heat is generated by BAT via non-shivering thermogenesis, which is the main defense our bodies employ against cold environments (Aherne and Hull, 1966). With increasing age, human brown adipose tissue (hBAT) was thought to diminish and ultimately vanish. It was not until his 1972 necropsy study that Heaton found human BAT persists throughout later stages of life, located at specific anatomical locations including around the kidneys, suprarenals and aorta, and in the neck and mediastinum (Heaton, 1972). In the 1980s, Huttunen et al. found that a large proportion of fat cells isolated from the neck contained potent mitochondrial enzymes in adult individuals exposed to cold (Huttunen et al., 1981). No active BAT had been identified in human adults prior to these discoveries because in vivo research into hBAT was severely hampered by a lack of methodologies that could accurately pinpoint its location.

In the mid-1990s, the emergence of positron emission tomography (PET) using the glucose tracer, 2-[18F]-fluoroD-2-deoxy-D-glucose (FDG), combined with computer tomography $(\mathrm{CT})$ renewed interest in hBAT and its role in energy expenditure (Cypess et al., 2009; Saito et al., 2009; van Marken Lichtenbelt et al., 2009). Just as in malignancy, hyper-metabolic hBAT has a high FDG uptake rate that can be detected by the minimally-invasive PET-CT technique in vivo. More recently, it has been discovered that a significant number of adult humans have active BAT that can be detected by PET-CT (Cypess et al., 2009; Saito et al., 2009; van Marken Lichtenbelt et al., 2009). However, PET-CT is not sensitive to BAT in the thermoneutral state because it depends on tissue glucose utilization (Chen et al., 2013). In recent years, magnetic resonance imaging (MRI) and functional (f)MRI, which detect changes associated with blood flow, have been used to assess hBAT volume and monitor responses to certain stimuli. MRI distinguishes between BAT and WAT according to three physiological criteria: BAT has a higher water-to-fat ratio, a higher density of mitochondria, and more blood vessels than WAT (Chen et al., 2013). According to imaging examinations, the main BAT depots are in the supraclavicular and neck regions with some additional paravertebral, mediastinal, para-aortic, and suprarenal localization (but not the interscapular region)
(Nedergaard et al., 2007), which coincide with former anatomical results.

Great advances about hBAT biology have emerged from imaging methods in recent years, and many of the achievements concur with those of murine BAT. Based on a higher FDG uptake, PET-CT scans showed that cold exposure significantly enhances hBAT activation (Saito et al., 2009; van Marken Lichtenbelt et al., 2009). Gender is another key determinant of BAT mass and activity. In general, women contain more BAT than men, while BAT distributions are similar across sexes (Cypess et al., 2009). Moreover, BAT volume and cold-activated FDG uptake are inversely correlated with age, beta-blocker use, body-mass index, body fat, and visceral fat (Nedergaard et al., 2007; Cypess et al., 2009; Saito et al., 2009). Thus, BAT is more abundant in younger and leaner individuals and decreased by long-term beta-blocker use. Altogether, these novel approaches have significantly advanced our understanding about hBAT localization and regulatory function and have provided researchers with the necessary tools to assess BAT's therapeutic potential in treating human obesity.

\section{MORPHOLOGICAL, FUNCTIONAL, AND GENETIC DIFFERENCES BETWEEN BAT AND WAT IN HUMANS}

Compared to WAT, human BAT is highly vascularized and contains more non-medullated nerve-fibers, which gives it a deeper color. BAT also plays an important role in energy dissipation, whereas the propensity to store energy in WAT is much higher since it can store excess calories as large, intracellular triacylglycerol (TG) droplets; the fat droplets from BAT on the other hand, are much smaller and contain numerous, similar-sized locules, which are conducive to rapid oxidation of lipids (Table 1). In the course of glucose and fatty acid catabolism, the BAT marker UCP1 mediates proton uncoupling over the inner mitochondrial membrane, thereby converting fuel substrates into heat instead of ATP, a process known as non-shivering thermogenesis (Daniel Ricquier, 1976; Nicholls and Locke, 1984). However, no UCP1 expression is detected in WAT (Virtanen et al., 2009). During cold exposure, non-shivering thermogenesis stabilizes core body temperature by consuming energy via oxidative metabolism. To maintain energy levels, the glucose uptake rate of hBAT is increased by a factor of 12 , while plasma insulin concentration is concurrently decreased and hWAT is unaffected (Orava et al., 2011). For comparison, insulin only enhances BAT glucose uptake 5-fold, whereas the effect on WAT is even smaller (Orava et al., 2011). The expression level of GLUT4, the insulin-responsive glucose transporter, is higher in BAT than in WAT, an observation that might underlie greater glucose uptake in BAT versus WAT (Virtanen et al., 2009). Interestingly, glucose can induce thermogenesis of hBAT in a sympathetic-independent manner (Lee et al., 2016). These results provoke the hypothesis 
Table 1. Differences between brown and white adipocytes

\begin{tabular}{|c|c|c|c|}
\hline & Brown & White & Ref. \\
\hline $\begin{array}{l}\text { Anatomical } \\
\text { location }\end{array}$ & $\begin{array}{l}\text { Thyroid, supraclavicular, mediastinal, } \\
\text { parathoracical, perirenal }\end{array}$ & $\begin{array}{l}\text { Subcutaneous, mesenteric, } \\
\text { retroperitoneal, omental }\end{array}$ & Cypess et al. (2009), Heaton (1972) \\
\hline $\begin{array}{l}\text { Cellular } \\
\text { morphology }\end{array}$ & Numerous, small lipid droplets & Unilocular, large lipid droplets & Heaton (1972) \\
\hline $\begin{array}{l}\text { Blood supply and } \\
\text { nerve-fibers }\end{array}$ & Abundant & Low & Heaton (1972), Hatai (1902) \\
\hline $\begin{array}{l}\text { Regulatory } \\
\text { function }\end{array}$ & $\begin{array}{l}\text { Dissipating energy as heat } \\
\text { production }\end{array}$ & Storing energy as triglycerides & Smith and Roberts (1964) \\
\hline Mitochondria & Abundant & Low & $\begin{array}{l}\text { Heaton (1972), Nicholls and Locke } \\
\text { (1984) }\end{array}$ \\
\hline UCP1 & High & Nearly undetectable & $\begin{array}{l}\text { Virtanen et al. (2009), Svensson } \\
\text { et al. (2011) }\end{array}$ \\
\hline Biomarkers & UCP1, LHX8, and ZIC1, & $\begin{array}{l}\text { MPZL2, HOXC9, EBF3, } \\
\text { FBXO31, and LEP, }\end{array}$ & Cypess et al. (2013) \\
\hline Insulin sensitivity & High & Low & Orava et al. (2011) \\
\hline Activators & Cold, exercise & High-calorie diet & $\begin{array}{l}\text { Saito et al. (2009), Orava et al. } \\
\text { (2011), Carriere et al. (2014) }\end{array}$ \\
\hline $\begin{array}{l}\text { Obesity and type } \\
2 \text { diabetes }\end{array}$ & Negative effect & Positive effect & Eger (1954), Himms-Hagen (1979) \\
\hline
\end{tabular}

that converting WAT into BAT is a promising approach to treat diabetes mellitus. However, the mechanisms of hBAT glucose uptake are not well understood. UCP1, the BAT marker gene, releases stored caloric energy as heat by uncoupling protons produced by oxidative phosphorylation (Cypess et al., 2009). The transcription factor PR domain containing 16 (PRDM16) is a critical switch factor in determining the commitment of mouse $\mathrm{Myf5}^{+}$precursors to brown adipocytes versus myogenic cells (Jimenez-Preitner et al., 2011; Kajimura et al., 2009). PRDM16 is more highly expressed in hBAT than hWAT. Similarly, other BAT-related mRNAs such as type 2 iodothyronine deiodinase (D2), peroxisome-proliferator-activated receptor $g$ coactivator $1 \alpha$ (PGC-1 $\alpha$ ), and $\beta 3$-adrenergic receptor also occur at significantly higher levels in hBAT versus hWAT (Virtanen et al., 2009). These results suggest that these genes play important roles in promoting BAT development, and their function in hBAT might emulate what is observed in mice.

\section{IS ADULT HUMAN BAT A CLASSICAL BAT OR BEIGE/BRITE ADIPOSE TISSUE?}

Two distinct types of UCP1-positive adipocytes have been identified in mice: the classical brown fat cells and brown-like fat cells (also called beige or brite adipocytes) (Ishibashi and Seale, 2010; Seale et al., 2008). Unlike classical brown fat, which is derived from a myf-5 lineage (Seale et al., 2008), beige cells in WAT are derived from a non-myf- 5 lineage and share features of both white and brown fat cells, including high expression of white adipocyte-specific markers (e.g.
aP2, adipsin, and PPAR-y) (Ishibashi and Seale, 2010). Adult hBAT, which is dispersed mainly in the supraclavicular, para-aortic, and suprarenal regions and is densely innervated, could be activated by cold through the sympathetic nervous system (Cannon and Nedergaard, 2011; Lowell and Spiegelman, 2000). However, unlike the classical brown adipocytes found in the interscapular regions of rodents and human infants, adult hBAT shares many molecular, histological, and functional characteristics with cold-induced beige fat found in the sWAT of rodents (Wu et al., 2012; Qiu et al., 2014). For example, mouse beige cells express Slc27a1, TMEM26, TBX1, CD40, and CD137 in a beigeselective manner, some of which, such as CD137, TMEM26, and TBX1, are highly expressed in adult hBAT (Wu et al., 2012), indicating that cold-induced hBAT is more closely related to rodent beige fat rather than classical brown fat. Within adult human neck BAT, the gene expression pattern of superficial neck fat resembles that of mouse sWAT, while deep neck fat is more like mouse BAT with high UCP1, PGC1- $\alpha$, and D2 levels (Quu et al., 2014). A whole-genome microarray experiment showed that the best molecular markers for grouping human fat cells are: 1) MPZL2, HOXC9, EBF3, FBXO31, and LEP, as WAT markers; 2) TNFRSF9, TMEM26, and SHOX2, as beige/brite markers; and 3) UCP1, LHX8, and ZIC1, as BAT markers (Table 1) (Cypess et al., 2013). Taken together, these studies suggest that cold-induced activation of hBAT primarily involves beige fat recruitment. However, more detailed work is needed to determine whether cold-induced activation of hBAT is mediated mainly by the sympathetic nervous system (SNS) 
or by alternative activation of the eosinophils-type 2 cytokine-macrophage signaling pathway (Qiu et al., 2014), or both.

\section{DISTINCTION OF HUMAN BAT AND MOUSE BAT}

Much of our knowledge about BAT is based on mouse studies and thus, it is of great interest to compare functionality, gene, and protein expression of mouse and human BAT. However, the principal rodent BAT depot lies in a welldefined anatomical location and is homogeneously composed of brown adipocytes, whereas hBAT is widely dispersed and occurs as a mixture of white, classical brown, and recruitable brite adipocytes (Jespersen et al., 2013) (Table 2). Comparison of microRNA expression profiles between mouse and human BAT reveals that while 145 miRNAs are expressed in both species, at least 250 other miRNAs are expressed exclusively in one species or the other (Guller et al., 2015). It is important to note too that the homology between mouse and human UCP1 is less than $80 \%$, suggesting there may be functional differences between species (Hughes et al., 2009). In addition, rodent UCP1 kinetics have been extensively studied in a variety of expression systems, whereas no corresponding study on human UCP1 kinetics has been reported to date (Hirschberg et al., 2011). Regarding thermogenic signal transduction, murine BAT is profoundly activated by selective $\beta 3$ adrenergic agonists (Feldmann et al., 2009). In human studies, treatment with the $\beta 3$ adrenergic agonist, mirabegron, or non-selective beta-blocker, propranolol, stimulated and impaired FDG uptake, respectively (Feldmann et al., 2009; van Baak et al., 2002). These reports suggest that $\beta 3$ adrenergic receptor signaling is capable of boosting hBAT metabolic activity. Nevertheless, there have been some conflicting reports suggesting the $\beta 3$ adrenergic response is very weak or non-responsive in hBAT; it has been suggested this is mainly due to sequence differences between the rodent and human receptors as well as low efficacy of the human $\beta 3$-adrenergic receptor in adipose tissue and poor drug bioavailability (van Baak et al., 2002) (Table 2). A very recent study also showed that glucocorticoids can acutely increase isoprenaline-stimulated respiration and UCP1 expression in human primary brown adipocytes, but substantially decrease isoprenaline-stimulated respiration and UCP1 in mouse primary brown adipocytes (Ramage et al., 2016) (Table 2), again suggesting the presence of speciesspecific differences between human and mouse BAT. More prospective studies would be of great value for future delineation of the difference between human and mouse BAT biology.

\section{MECHANISTIC EFFECTS OF AMBIENT TEMPERATURE ON HBAT}

Cold exposure profoundly enhances hBAT perfusion and activity, and improves glucose and fatty acid uptake, thereby supporting cold-induced energy dissipation (Saito et al., 2009; Orava et al., 2011). These observations reveal hBAT as an attractive target for treating and preventing obesity. However, the promoting effect of cold-exposure on energy expenditure is markedly suppressed by fasting-induced insulin resistance (Hanssen et al., 2015). Rodents studies show that the stimulatory effects of cold exposure are emulated by electrical stimulation of sympathetic nerves within BAT and by administration of adrenergic agonists, but abolished by surgical severing of the sympathetic nerves or adrenergic blocker treatment (Shimizu et al., 1991), indicating that cold-exposure stimulates BAT via sympathetic nerves. However, the mechanism by which the SNS regulates hBAT upon cold-exposure is still unknown. Agents that activate the SNS failed to activate BAT in human studies, presumably due to the systemic effects of SNS agonists and minimal BAT responsiveness (Cypess et al., 2012). Epinephrine, a sympathomimetic drug, does not activate hBAT at a dose that leads to broad activation of the SNS (Cypess et al., 2012), suggesting that cold-exposure activates specific sympathetic pathways responsible for hBAT

Table 2. Differences between human and mouse adipocytes

\begin{tabular}{|c|c|c|c|}
\hline & Human BAT & Murine BAT & Ref. \\
\hline Distribution & Dispersed & $\begin{array}{l}\text { The main BAT depots are in } \\
\text { well-defined anatomic sites }\end{array}$ & $\begin{array}{l}\text { Heaton (1972), Jespersen et al. } \\
\text { (2013) }\end{array}$ \\
\hline Cell composition & $\begin{array}{l}\text { A mix of white, classical brown, } \\
\text { and recruitable brite adipocytes }\end{array}$ & $\begin{array}{l}\text { Homogeneously composed of } \\
\text { brown adipocytes }\end{array}$ & $\begin{array}{l}\text { Chen et al. (2013), Jespersen et al. } \\
\text { (2013) }\end{array}$ \\
\hline $\begin{array}{l}\text { MicroRNA } \\
\text { expression }\end{array}$ & \multicolumn{2}{|c|}{$\begin{array}{l}145 \text { miRNAs are expressed in BAT of both species while expression of } \\
\text { at least } 250 \text { other miRNAs is exclusive to one species or the other }\end{array}$} & Guller et al. (2015) \\
\hline $\begin{array}{l}\text { Response to } \beta 3 \\
\text { adrenergic } \\
\text { agonists }\end{array}$ & Weak & Strong & $\begin{array}{l}\text { Feldmann et al. (2009), van Baak } \\
\text { et al. (2002) }\end{array}$ \\
\hline $\begin{array}{l}\text { Changes with } \\
\text { increasing age }\end{array}$ & Gradually decline & Stably exist & $\begin{array}{l}\text { Heaton (1972), Smith and Roberts } \\
\text { (1964) }\end{array}$ \\
\hline $\begin{array}{l}\text { Glucocorticoid } \\
\text { effects }\end{array}$ & $\begin{array}{l}\text { Acutely increase but chronically } \\
\text { suppress BAT activity }\end{array}$ & $\begin{array}{l}\text { Substantially suppress BAT } \\
\text { activity }\end{array}$ & $\begin{array}{l}\text { Ramage et al. (2016), Zilberfarb } \\
\text { et al. (2001), Barclay et al. (2015) }\end{array}$ \\
\hline
\end{tabular}


activation. In addition, most known effectors of UCP1-dependent thermogenesis such as certain neuropeptides, leptin, and capsaicin all converge on SNS activation. One of the major drawbacks with using broad sympathetic activation to stimulate thermogenesis however, are the serious-side effects and increased risk for heart attack (Bonet et al., 2013). Although cold exposure profoundly increases plasma concentrations of SNS activation biomarkers like norepinephrine and the secondary messenger, cyclic adenosine monophosphate (CAMP), this effect has been found in humans both with and without cold-activated BAT (Orava et al., 2011). In addition, stimulation of the parasympathetic vagus nerve also increases energy expenditure in human BAT, although the mechanism is unclear (Vijgen et al., 2013). These reports suggest that hBAT stimulation through generalized sympathomimetic drugs is not a viable approach and thus, more extensive analyses in human BAT are needed.

Cold exposure may also alter human physiology by crosstalking with immune cells to release cytokines capable of inducing fat browning. In mice, cold exposure rapidly promoted eosinophil-dependent and interleukin 4 (IL-4)-stimulated alternative activation of adipose macrophages, which secrete catecholamines to induce thermogenic gene expression and/or lipolysis in both BAT and WAT (Qiu et al., 2014; Nguyen et al., 2011). ILC2s can promote the eosinophi//L-4Ra/alternatively-activated macrophage (AAMac) (Qiu et al., 2014; Liu et al., 2014; Wu et al., 2011). Besides, ILC2s have been shown to promote beiging in an IL33-depend pathway in part via production of enkephalin peptides that elicit beiging (Brestoff et al., 2015). IL-4 stimulation of human monocytes has also been reported to promote alternative activation, catecholamine production, lipolytic activity of adipocytes, and dysregulated ILC2 responses in WAT are a conserved feature of obesity in humans and mice (Brestoff et al., 2015), suggesting these pathways may play the same roles across species (Nguyen et al., 2011). In humans, mild cold exposure increases plasma levels of fibroblast growth factor-21 (FGF-21) (Lee et al., 2013), a hormone that promotes systemic glucose uptake and oxidation. In hBAT, FGF-21 expression positively correlates with UCP1 expression, promotes BAT activity and recruits inducible brown adipocytes via autocrine and/or endocrine mechanisms (Hondares et al., 2014). Future studies aiming to identify secreted factors that promote thermogenesis and/ or beiging will undoubtedly advance our understanding about thermogenic regulation and may lead to novel antiobesity drug treatments.

\section{EFFECTS OF EXERCISE ON HBAT ACTIVITY AND THE UNDERLYING MECHANISMS}

Exercise could produce energetic benefits much greater than the direct calories cost (Speakman and Selman, 2003). Irisin, a myokine that upregulates human brown adipocyte gene expression and thermogenesis in human neck

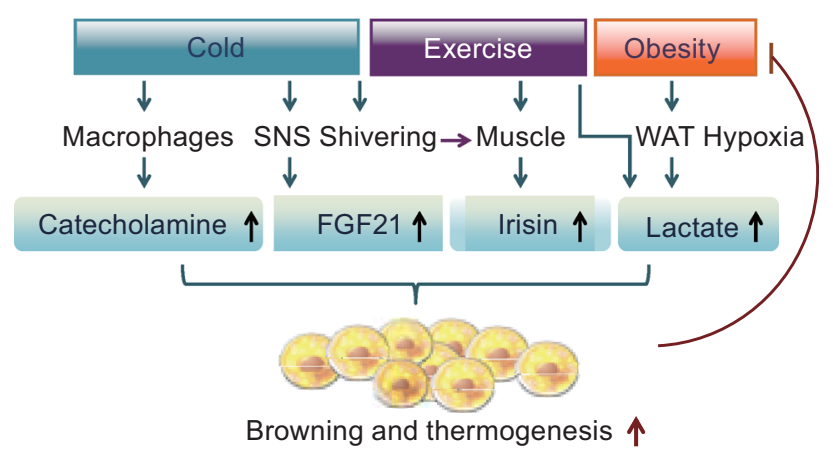

Figure 1. The mechanisms by which cold, exercise, and obesity regulate thermogenesis. Cold exposure could improve browning and thermogenesis of human adipocytes through macrophages, sympathetic nervous system (SNS), and muscle contraction. Cold exposure rapidly promoted alternative activation of adipose tissue resident macrophages, leading to the secretion of catecholamines to induce beige fat development and thermogenesis. Cold activates the SNS, leading to the upregulation of fibroblast growth factor 21 (FGF21). Cold and exercise cause muscle contraction, which stimulates irisin secretion and induces shivering thermogenesis. In response to cold-exposure, the shivering-induced irisin promotes browning and thermogenesis of human adipocytes in concert with the SNS-induced FGF21. Exercise is also able to promote browning and thermogenesis by increasing lactate production. In obese individuals, enhanced lactate in adipocytes promotes browning and thermogenesis of neighboring adipocytes, which in turn, limits the accumulation of fat, thereby impeding the development of obesity.

adipocytes, was found to be enhanced by exercise in humans (Bostrom et al., 2012; Lee et al., 2014). Interestingly, cold-induced shivering by muscle contraction stimulates irisin secretion to levels that are nearly comparable to those induced by exercise (Lee et al., 2014), suggesting that irisin may link exercise to cold-induced thermogenesis and promote browning of human neck adipocytes and thermogenesis in concert with FGF21 during cold-exposure (Lee et al., 2014) (Fig. 1). However, another study showed that neither acute nor regular exercise altered irisin levels, although the intensity of habitual physical activity, muscle volume, strength, contractility are positively associated with circulating irisin (Kurdiova et al., 2014). Thus, it remains to be further determined as to whether irisin regulates thermogenesis in humans. Besides irisin, increased plasma lactate concentrations resulting from strenuous exercise may also contribute to browning. Lactate, which is a glycolytic waste product, drives the browning of human white adipocytes through drastic and rapid inductions of several brown-adipocyte genes including UCP1 via peroxisome proliferator 
activated receptor- $($ (PPAR- $ү$ ), a master transcription factor regulating adipocyte differentiation (Carriere et al., 2014). Furthermore, obesity elevates lactate production in rat WAT due to hypoxia (DiGirolamo et al., 1992). The lactate then stimulates "browning" of neighboring white adipocytes through an autocrine/paracrine effect. This could reflect a self-regulatory response to limit the accumulation of fat during obesity (Trayhurn and Alomar, 2015). Indeed, fat cells cultured from obese or diabetic humans metabolize more lactate than lean individuals (DiGirolamo et al., 1992), which indicates that lactate might play the same role in vivo. Human natriuretic peptides (NPs) also play a physiological role in exercise-induced lipolysis (Moro et al., 2008). In human adipocytes, atrial natriuretic peptide (ANP) and brain natriuretic peptide (BNP) induce a brown-like adaptive thermogenic response by activating PPARy coactivator-1a (PGC-1 $\alpha$ ) and UCP1 expression and stimulating mitochondrial biogenesis, thereby increasing uncoupled and total respiration (Bordicchia et al., 2012). Beyond the calories burned at the gym, it is becoming increasingly clear that changes in both gene expression and the secretome are likely responsible for at least some of the beneficial effects of exercise on whole body energy expenditure (Speakman and Selman, 2003).

\section{REGULATORY FACTORS OF HUMAN BAT}

Human brown adipocytes are regulated by many intrinsic factors, including glucocorticoids and bile acids. In the absence of glucocorticoids, brown adipocytes differentiate poorly, particularly during early differentiation. Glucocorticoids induce PPAR-y and CCAAT/enhancer binding protein- $\beta(C / E B P-\beta)$ expression as well as increase PGC1- $\alpha$ and GLUT-4 levels, while reducing the expression of DNA binding-2 inhibitor (Id2) and TNF- $\alpha$ (Ramage et al., 2016; Zilberfarb et al., 2001). Acute treatment of human brown adipocytes with glucocorticoids at physiological concentrations increased basal and adrenergic stimulated respiration and UCP1 expression (Ramage et al., 2016). However, chronic glucocorticoid stimulation also suppressed $\beta$-adrenergic thermogenesis in hBAT (Ramage et al., 2016; Barclay et al., 2015), which may underlie glucocorticoid therapy-associated body-weight gain. The bile acid, chenodeoxycholic acid (CDCA), is another steroid molecule that is essential for metabolism and thought to increase human whole-body energy expenditure. In human brown adipocytes, CDCA improves mitochondrial uncoupling and D2 expression via a $G$ protein-coupled receptor TGR5 (G-protein coupled receptor for bile acids)-dependent mechanism (Broeders et al., 2015). As a registered and generally recognized safe drug for humans (Broeders et al., 2015), CDCA is a promising candidate to activate hBAT and counter obesity and its related metabolic diseases. Thus, identification of more endogenous regulatory factors is critically needed to elucidate hBAT biology and the body's complex responses to regulate energy expenditure.

\section{DEVELOPMENT OF IMMORTALIZED HUMAN BROWN ADIPOCYTES}

Despite advances in our understanding of murine BAT, there remain numerous knowledge gaps concerning the biological mechanisms of hBAT. Although thermal imaging and FDG/ MRI scans are capable of capturing human BAT activity noninvasively, for in vitro research, human cell lines are still needed to uncover the genetic, pharmacological, and environmental determinants of BAT biological functions. PAZ6, the first available immortalized human BAT cell line (Zilberfarb et al., 1997), has been used to study generic cellular processes and evaluate the expression of adipogenic markers. Differentiated PAZ6 adipocytes express a myriad of brown adipocyte markers including $\beta 1, \beta 2$, and $\beta 3$ adrenergic receptors ( $\beta-A R), \alpha 2 A-A R$, lipoprotein lipase, hormone sensitive lipase, adipsin, the glucose transporters Glut 1 and Glut 4, leptin, and UCP1 (Zilberfarb et al., 1997). Among these BAT markers, the expression of leptin and GLUT1 is markedly stimulated by hypoxia (Grosfeld et al., 2002), which could be an adaptive mechanism that promotes angiogenesis and increases adipose tissue oxygenation (Grosfeld et al., 2002). While it is widely accepted that the rodent $\beta 3$-adrenoceptor is essential for regulating fat metabolism, its role in human BAT is still under debate (Arch and Wilson, 1996). According to a saturation binding analysis using PAZ6 adipocytes, the $\beta 3-A R$ appears to be the most abundant $\beta$-AR subtype. The selective $\beta 3$-AR agonist, CGP 12177A, markedly increases CAMP concentration and lipolysis activity in PAZ6 adipocytes (Zilberfarb et al., 1997). Pretreating PAZ6 adipocytes with norepinephrine however, down-regulates $\beta$-AR number and desensitizes $\beta 3-A R$ signaling (Jockers et al., 1998). These results suggest that $\beta 3$ AR in human brown adipocyte is functionally coupled to lipolysis (Jockers et al., 1998). Thiazolidinediones are a popular class of drugs used to treat type 2 diabetes because of their ability to increase insulin sensitivity by binding to PPAR- $y$, which alters the expression of genes involved in glucose and lipid metabolism. PAZ6 cells treated with thiazolidinediones exhibited enhanced brown adipocyte differentiation as demonstrated by increased triglyceride content and adipocyte-specific gene expression, including aP2, PPAR-y, B3-AR, HSL, and UCP2 (Strobel et al., 1999). Other small molecules thought to promote hBAT function such as dehydroepiandrosterone (DHEA) and retinoic acid (RA) have also been tested in PAZ6 cells. Serum concentrations of DHEA, a steroid sex hormone precursor, inversely correlate with biomarkers of metabolic syndrome, indicating that DHEA may modulate adipose tissue mass and function. DHEA inhibits PAZ6 preadipocyte proliferation by blocking the $G_{1} / S$ or $G_{2} / M$ transition. By contrast, UCP1 mRNA levels are higher in PAZ6 cells differentiated in the presence of 
DHEA. The inhibitory effect on PAZ6 pre-adipocyte cell cycle progression and the promoting effect on PAZ6 adipocyte gene expression may represent a pro-adipogenic mechanism (Rice et al., 2010). In PAZ6 adipocytes, retinoic acid increases UCP1 gene expression in a PGC1a-dependent way, while the PPARa-specific agonist, WY14643, failed to regulate UCP1 gene expression without RA (Oberkofler et al., 2002). However, the mechanism by which RA regulates hBAT in vivo remains uncertain.

\section{SUMMARY AND FUTURE PERSPECTIVES}

The therapeutic potential of hBAT as an anti-glycemic, antilipidemic, and weight loss-inducing 'metabolic panacea' is postulated by calculations showing that when fully activated, 63 grams of BAT would burn the energy-equivalence of 4.1 kilograms of WAT over the course of a year (Virtanen et al., 2009). Considering its striking capacity for energy dissipation, the findings in hBAT are clinically valuable for guiding future prevention strategies and treatments for obesity, insulin resistance, and other related metabolic diseases (Hall et al., 2011). Increasing human energy expenditure through hBAT by cold-exposure (Cypess et al., 2009; Aherne and Hull, 1966) and exercise (Lee et al., 2013; Hondares et al., 2014) however, would require drastic changes in life style. Considering the discomfort of cold exposure, exercise is likely the preferred approach for enhancing hBAT activity. The increased irisin and lactate concentrations that are induced during exercise have been reported to promote adipocyte browning remodeling and are potential approaches for treating obesity without the exercise (Bostrom et al., 2012; Carriere et al., 2014). Molecules such as FGF-21, natriuretic peptides, and thyroid hormones have also been suggested as potential drugs to counteract obesity and its related metabolic diseases. The thyroid hormone, triiodothyronine (T3), has been shown to profoundly promote mitochondrial biogenesis and UCP1 expression in brownlike fat cells as well as skeletal muscle and brown adipose tissue (Crisan et al., 2008). Another anti-obesity strategy that has not been considered in this review, but warrant serious investigation, includes transforming muscles cells into brown fat. Interestingly, a stationary population of human skeletal muscle cells expressing the CD34 surface protein have been found to differentiate in vitro into genuine brown adipocytes with a high level of UCP1 expression (Crisan et al., 2008), suggesting that human skeletal muscle cells have the potential to transdifferentiate into brown adipocytes. Thus, finding new ways to reprogram skeletal muscle into brown fat represents another novel approach to promote thermogenesis and combat obesity.

Although pharmacological means to treat obesity are possible, supraphysiological concentrations of these medications are associated with severe side effects. In addition, only a few defects in human BAT development and thermogenesis due to gene mutations have been described
(Yoneshiro et al., 2005) thus, a better understanding of the physiological processes underlying hBAT is still required before BAT-related therapies can be developed. At present, much of our knowledge about BAT is based on mouse studies and hBAT research is still limited by a number of factors such as access to high-quality, ethically-approved clinical samples, reliable methods for measuring BAT volume and activity, and better ways to stimulate BAT function with minimal adverse effects. One protocol, which collects both MRI and 18F-FDG PET-CT scans, has been reported to give accurate estimates of hBAT imaging volumes (Gifford et al., 2015). In the future, this approach could be used in longitudinal studies to assess therapeutic interventions. In conclusion, we opine that a better understanding of hBAT physiology will lead to new pharmacological targets for treating obesity, and given the current obesity epidemic, more physiological studies of brown adipose tissue in healthy adult individuals are needed to fully characterize its regulatory function and therapeutic potential.

\section{ACKNOWLEDGEMENTS}

We thank Dr. Hong Ruan for initial English-editing assistance. This work was supported by grants from the National Basic Research Program (973 Program) (No. 2014CB910501), the National Natural Science Foundation of China (Grant No. 81000316), and a grant from the NIH (DK76902).

\section{ABBREVIATIONS}

ANP, atrial natriuretic peptide; BAT, brown adipose tissue; BNP, brain natriuretic peptide; cAMP, cyclic adenosine monophosphate; CDCA, chenodeoxycholic acid; C/EBP- $\beta$, CCAAT/enhancer binding protein- $\beta$; $C T$, computer tomography; $D 2$, type 2 iodothyronine deiodinase; DHEA, dehydroepiandrosterone; FDG, 2-[18F]-fluoro-D2-deoxy-D-glucose; FGF-21, fibroblast growth factor-21; hBAT, human brown adipose tissue; IL-4, interleukin 4; MRI, magnetic resonance imaging; NPs, natriuretic peptides; PET, positron emission tomography; PGC-1 $\alpha$, peroxisome-proliferator-activated receptor g coactivator $1 \alpha$; PPAR- $\gamma$, peroxisome proliferator activated receptor-y; PRDM16, PR domain containing 16; RA, retinoic acid; SNS, sympathetic nervous system; T3, triiodothyronine; TG, triacylglycerol; WAT, white adipose tissue; WHO, World Health Organization.

\section{COMPLIANCE WITH ETHICS GUIDELINES}

No potential conflicts of interest were declared by the authors. This article does not contain any studies with human or animal subjects performed by the any of the authors.

\section{AUTHOR CONTRIBUTIONS}

X.L. wrote manuscript, C.C. discussed and edited the manuscript, and F.L. contributed to discussion, reviewing, and editing of the manuscript. 


\section{OPEN ACCESS}

This article is distributed under the terms of the Creative Commons Attribution 4.0 International License (http://creativecommons.org/ licenses/by/4.0/), which permits unrestricted use, distribution, and reproduction in any medium, provided you give appropriate credit to the original author(s) and the source, provide a link to the Creative Commons license, and indicate if changes were made.

\section{REFERENCES}

Aherne W, Hull D (1966) Brown adipose tissue and heat production in the newborn infant. J Pathol Bacteriol 91:223-234

Arch JR, Wilson S (1996) Beta 3-adrenoceptors and the regulation of metabolism in adipose tissues. Biochem Soc Trans 24:412418

Barclay JL, Agada H, Jang C, Ward M, Wetzig N, Ho KK (2015) Effects of glucocorticoids on human brown adipocytes. J Endocrinol 224:139-147

Bonet ML, Oliver P, Palou A (2013) Pharmacological and nutritional agents promoting browning of white adipose tissue. Biochim Biophys Acta 1831:969-985

Bordicchia M, Liu D, Amri EZ et al (2012) Cardiac natriuretic peptides act via p38 MAPK to induce the brown fat thermogenic program in mouse and human adipocytes. J Clin Investig 122:1022-1036

Bostrom P, Wu J, Jedrychowski MP et al (2012) A PGC1-alphadependent myokine that drives brown-fat-like development of white fat and thermogenesis. Nature 481:463-468

Brestoff JR, Kim BS, Saenz SA et al (2015) Group 2 innate lymphoid cells promote beiging of white adipose tissue and limit obesity. Nature 519:242-246

Broeders EP, Nascimento EB, Havekes B et al (2015) The bile acid chenodeoxycholic acid increases human brown adipose tissue activity. Cell Metab 22:418-426

Cannon B, Nedergaard J (2011) Nonshivering thermogenesis and its adequate measurement in metabolic studies. J Exper Biol 214:242-253

Carriere A, Jeanson Y, Berger-Muller S et al (2014) Browning of white adipose cells by intermediate metabolites: an adaptive mechanism to alleviate redox pressure. Diabetes 63:3253-3265

Chen YC, Cypess AM, Chen YC et al (2013) Measurement of human brown adipose tissue volume and activity using anatomic MR imaging and functional MR imaging. J Nucl Med 54:1584-1587

Crisan M, Casteilla L, Lehr L et al (2008) A reservoir of brown adipocyte progenitors in human skeletal muscle. Stem Cells 26:2425-2433

Cypess AM, Lehman S, Williams $G$ et al (2009) Identification and importance of brown adipose tissue in adult humans. $\mathrm{N}$ Engl $\mathrm{J}$ Med 360:1509-1517

Cypess AM, Chen YC, Sze C et al (2012) Cold but not sympathomimetics activates human brown adipose tissue in vivo. Proc Natl Acad Sci USA 109:10001-10005

Cypess AM, White AP, Vernochet C et al (2013) Anatomical localization, gene expression profiling and functional characterization of adult human neck brown fat. Nat Med 19:635-639
Daniel Ricquier J-CK (1976) Mitochondrial protein alterations in active brown fat: a sodium dodecyl sulfate-polyacrylamide gel electrophoretic study. Biochem Biophys Res Commun 73:577583

DiGirolamo M, Newby FD, Lovejoy J (1992) Lactate production in adipose tissue: a regulated function with extra-adipose implications. FASEB J 6:2405-2412

Eckel RH, Alberti KG, Grundy SM, Zimmet PZ (2010) The metabolic syndrome. Lancet 375:181-183

Eger W (1954) The significance of fatty tissue in obesity. I. On the histology, physiology and pathology of the white and brown fatty tissue. Die Med 20:701-704

Feldmann HM, Golozoubova V, Cannon B, Nedergaard J (2009) UCP1 ablation induces obesity and abolishes diet-induced thermogenesis in mice exempt from thermal stress by living at thermoneutrality. Cell Metab 9:203-209

Gifford A, Towse TF, Walker RC, Avison MJ, Welch EB (2015) Human brown adipose tissue depots automatically segmented by positron emission tomography/computed tomography and registered magnetic resonance images. J Vis Exp 96:e52415

Grosfeld A, Zilberfarb V, Turban S, Andre J, Guerre-Millo M, Issad T (2002) Hypoxia increases leptin expression in human PAZ6 adipose cells. Diabetologia 45:527-530

Guller I, McNaughton S, Crowley T et al (2015) Comparative analysis of microRNA expression in mouse and human brown adipose tissue. BMC Genom 16:820

Hall KD, Sacks G, Chandramohan D et al (2011) Quantification of the effect of energy imbalance on bodyweight. Lancet 378:826837

Hanssen MJ, Wierts R, Hoeks J et al (2015) Glucose uptake in human brown adipose tissue is impaired upon fasting-induced insulin resistance. Diabetologia 58:586-595

Hatai $S$ (1902) On the presence in human embryos of an interscapular gland corresponding to the so-called hibernating gland of lower mammals. Anat Anz 21:369-373

Heaton JM (1972) The distribution of brown adipose tissue in the human. J Anat 112:35-39

Himms-Hagen J (1979) Obesity may be due to a malfunctioning of brown fat. Can Med Assoc J 121:1361-1364

Hirschberg V, Fromme T, Klingenspor M (2011) Test systems to study the structure and function of uncoupling protein 1: a critical overview. Front Endocrinol 2:63

Hondares E, Gallego-Escuredo JM, Flachs P et al (2014) Fibroblast growth factor-21 is expressed in neonatal and pheochromocytoma-induced adult human brown adipose tissue. Metabolism 63:312-317

Hughes DA, Jastroch M, Stoneking M, Klingenspor M (2009) Molecular evolution of UCP1 and the evolutionary history of mammalian non-shivering thermogenesis. BMC Evol Biol 9:4

Huttunen P, Hirvonen J, Kinnula V (1981) The occurrence of brown adipose tissue in outdoor workers. Eur J Appl Physiol 46:339-345

Ishibashi J, Seale P (2010) Medicine. Beige can be slimming. Science 328:1113-1114

Jespersen NZ, Larsen TJ, Peijs L et al (2013) A classical brown adipose tissue mRNA signature partly overlaps with brite in the supraclavicular region of adult humans. Cell Metab 17:798-805 
Jimenez-Preitner M, Berney X, Uldry M et al (2011) Plac8 is an inducer of C/EBPbeta required for brown fat differentiation, thermoregulation, and control of body weight. Cell Metab 14:658-670

Jockers R, Issad T, Zilberfarb V, de Coppet P, Marullo S, Strosberg $A D$ (1998) Desensitization of the beta-adrenergic response in human brown adipocytes. Endocrinology 139:2676-2684

Kajimura S, Seale P, Kubota K et al (2009) Initiation of myoblast to brown fat switch by a PRDM16-C/EBP-beta transcriptional complex. Nature 460:1154-1158

Kurdiova T, Balaz M, Vician M et al (2014) Effects of obesity, diabetes and exercise on Fndc5 gene expression and irisin release in human skeletal muscle and adipose tissue: in vivo and in vitro studies. J Physiol 592:1091-1107

Lee P, Brychta RJ, Linderman J, Smith S, Chen KY, Celi FS (2013) Mild cold exposure modulates fibroblast growth factor 21 (FGF21) diurnal rhythm in humans: relationship between FGF21 levels, lipolysis, and cold-induced thermogenesis. J Clin Endocrinol Metab 98:E98-E102

Lee P, Linderman JD, Smith S et al (2014) Irisin and FGF21 are coldinduced endocrine activators of brown fat function in humans. Cell Metab 19:302-309

Lee P, Bova R, Schofield L et al (2016) Brown adipose tissue exhibits a glucose-responsive thermogenic biorhythm in humans. Cell Metab 23:602-609

Liu PS, Lin YW, Lee B, McCrady-Spitzer SK, Levine JA, Wei LN (2014) Reducing RIP140 expression in macrophage alters ATM infiltration, facilitates white adipose tissue browning, and prevents high-fat diet-induced insulin resistance. Diabetes 63:4021-4031

Lowell BB, Spiegelman BM (2000) Towards a molecular understanding of adaptive thermogenesis. Nature 404:652-660

Moro C, Pillard F, de Glisezinski I et al (2008) Exercise-induced lipid mobilization in subcutaneous adipose tissue is mainly related to natriuretic peptides in overweight men. Am J Physiol Endocrinol Metab 295:E505-E513

Nedergaard J, Bengtsson T, Cannon B (2007) Unexpected evidence for active brown adipose tissue in adult humans. Am J Physiol Endocrinol Metab 293:E444-E452

Nguyen KD, Qiu Y, Cui X et al (2011) Alternatively activated macrophages produce catecholamines to sustain adaptive thermogenesis. Nature 480:104-108

Nicholls DG, Locke RM (1984) Thermogenic mechanisms in brown fat. Physiol Rev 64:1-64

Oberkofler H, Esterbauer H, Linnemayr V, Strosberg AD, Krempler F, Patsch W (2002) Peroxisome proliferator-activated receptor (PPAR) gamma coactivator-1 recruitment regulates PPAR subtype specificity. J Biol Chem 277:16750-16757

Orava J, Nuutila P, Lidell ME et al (2011) Different metabolic responses of human brown adipose tissue to activation by cold and insulin. Cell Metab 14:272-279

Qiu Y, Nguyen KD, Odegaard Jl et al (2014) Eosinophils and type 2 cytokine signaling in macrophages orchestrate development of functional beige fat. Cell 157:1292-1308

Ramage LE, Akyol M, Fletcher AM et al (2016) Glucocorticoids acutely increase brown adipose tissue activity in humans, revealing species-specific differences in UCP-1 regulation. Cell Metab 24:130-141
Rice SP, Zhang L, Grennan-Jones F et al (2010) Dehydroepiandrosterone (DHEA) treatment in vitro inhibits adipogenesis in human omental but not subcutaneous adipose tissue. Mol Cell Endocrinol 320:51-57

Saito M, Okamatsu-Ogura Y, Matsushita M et al (2009) High incidence of metabolically active brown adipose tissue in healthy adult humans: effects of cold exposure and adiposity. Diabetes 58:1526-1531

Seale P, Bjork B, Yang W et al (2008) PRDM16 controls a brown fat/ skeletal muscle switch. Nature 454:961-967

Shimizu Y, Nikami H, Saito M (1991) Sympathetic activation of glucose utilization in brown adipose tissue in rats. J Biochem 110:688-692

Smith RE, Roberts JC (1964) Thermogenesis of brown adipose tissue in cold-acclimated rats. Am J Physiol 206:143-148

Speakman JR, Selman C (2003) Physical activity and resting metabolic rate. Proc Nutr Soc 62:621-634

Strobel A, Siquier K, Zilberfarb V, Strosberg AD, Issad T (1999) Effect of thiazolidinediones on expression of UCP2 and adipocyte markers in human PAZ6 adipocytes. Diabetologia 42:527-533

Svensson PA, Jernas M, Sjoholm K et al (2011) Gene expression in human brown adipose tissue. Int J Mol Med 27:227-232

Trayhurn P, Alomar SY (2015) Oxygen deprivation and the cellular response to hypoxia in adipocytes: perspectives on white and brown adipose tissues in obesity. Front Endocrinol 6:19

van Baak MA, Hul GB, Toubro S et al (2002) Acute effect of L-796568, a novel beta 3-adrenergic receptor agonist, on energy expenditure in obese men. Clin Pharmacol Ther 71:272-279

van Marken Lichtenbelt WD, Vanhommerig JW, Smulders NM et al (2009) Cold-activated brown adipose tissue in healthy men. N Engl J Med 360:1500-1508

Vijgen GH, Bouvy ND, Leenen L et al (2013) Vagus nerve stimulation increases energy expenditure: relation to brown adipose tissue activity. PLoS ONE 8:e77221

Virtanen KA, Lidell ME, Orava $\mathrm{J}$ et al (2009) Functional brown adipose tissue in healthy adults. N Engl J Med 360:1518-1525

World Health Organization Fact Sheets: Obesity and Overweight (2016) (article online). http://www.who.int/mediacentre/factsheets/ fs311/en/

Wu D, Molofsky AB, Liang HE et al (2011) Eosinophils sustain adipose alternatively activated macrophages associated with glucose homeostasis. Science 332:243-247

Wu J, Bostrom P, Sparks LM et al (2012) Beige adipocytes are a distinct type of thermogenic fat cell in mouse and human. Cell 150:366-376

Yoneshiro T, Ogawa T, Okamoto N et al (2005) Impact of UCP1 and beta3AR gene polymorphisms on age-related changes in brown adipose tissue and adiposity in humans. Int J Obes 2013 (37):993-998

Zilberfarb V, Pietri-Rouxel F, Jockers R et al (1997) Human immortalized brown adipocytes express functional beta3-adrenoceptor coupled to lipolysis. J Cell Sci 110(Pt 7):801-807

Zilberfarb V, Siquier K, Strosberg AD, Issad T (2001) Effect of dexamethasone on adipocyte differentiation markers and tumour necrosis factor-alpha expression in human PAZ6 cells. Diabetologia 44:377-386 